\title{
Unitary Long-Time Evolution with Quantum Renormalization Groups and Artificial Neural Networks
}

\author{
Heiko Burau* and Markus Heyl \\ Max-Planck-Institut für Physik Komplexer Systeme, Nöthnitzer Straße 38, 01187 Dresden, Germany
}

(Received 1 October 2020; revised 12 April 2021; accepted 10 June 2021; published 27 July 2021)

\begin{abstract}
In this work, we combine quantum renormalization group approaches with deep artificial neural networks for the description of the real-time evolution in strongly disordered quantum matter. We find that this allows us to accurately compute the long-time coherent dynamics of large many-body localized systems in nonperturbative regimes including the effects of many-body resonances. Concretely, we use this approach to describe the spatiotemporal buildup of many-body localized spin-glass order in random Ising chains. We observe a fundamental difference to a noninteracting Anderson insulating Ising chain, where the order only develops over a finite spatial range. We further apply the approach to strongly disordered twodimensional Ising models, highlighting that our method can be used also for the description of the real-time dynamics of nonergodic quantum matter in a general context.
\end{abstract}

DOI: 10.1103/PhysRevLett.127.050601

Introduction.-The understanding of emergent behavior in quantum many-body systems is largely based on the discovery of effective descriptions of analytically unsolvable models [1]. An essential toolkit to find the former constitutes renormalization group $(\mathrm{RG})$ methods. They are traditionally applied on systems in thermal equilibrium, thereby explaining many collective phenomena including structured phases, phase transitions, critical scaling, and universality. In the past decade, real-space RGs have been developed that also aim to explain analogs of these wellknown phenomena in systems where a thermodynamic treatment breaks down due to strong quenched disorder [2-10].

Whereas real-space RGs successfully operate in the stationary setting at the level of individual eigenstates [11-16], reaching a quantitative description of the dynamical properties of quantum many-body systems appears even more challenging. So far, coherent dynamics of quantum matter far from equilibrium has been mostly simulated using tensor networks methods [17-24] or exact diagonalization [25-28], with recent developments using machine learning methods and restricted Boltzmann machines (RBMs) [29] or, more generally, artificial neural networks (ANNs) [29-31]. Still, accessing quantitatively the longtime dynamics for large quantum many-body systems,

Published by the American Physical Society under the terms of the Creative Commons Attribution 4.0 International license. Further distribution of this work must maintain attribution to the author(s) and the published article's title, journal citation, and DOI. Open access publication funded by the Max Planck Society. especially in spatial dimensions beyond one, represents a major challenge [32-34].

In this work, we show how ANNs can be utilized in a different way for numerically exactly time-integrating effective descriptions of generically interacting systems generated by RG methods. As a concrete example, we explore the temporal buildup of many-body localized (MBL) spin-glass order out of a simple polarized state for a large, disordered spin chain [see Fig. 1(b)], among other long-time dynamics in one-dimensional (1D) and twodimensional (2D) lattices. We begin by formulating a prototypical strong-disorder RG (SDRG) for spin-1/2 systems of arbitrary spatial dimension and map its transformations into the time domain. As a result, we obtain a quantum circuit [see Fig. 1(a)] as an effective description of the time-evolution operator. Hereafter, we show that this circuit can be encoded efficiently into deep ANNs associated with typical initial conditions for quantum real-time dynamics. This allows us to quantitatively represent time-evolved many-body quantum states not only at short but also long times. We note that our method avoids a discretization of time but relies on a renormalized Hamiltonian that is assumed to effectively describe the relevant physics up to some finite but nevertheless long timescale.

The scheme we introduce in the following can be applied for any generic, but strongly disordered, spin- $1 / 2$ system. Concretely, we will apply it to a paradigmatic interacting disordered quantum Ising model [35] of the form

$$
H=\sum_{\langle i j\rangle} J_{i j} \sigma_{i}^{z} \sigma_{j}^{z}+J_{i j}^{(x)} \sigma_{i}^{x} \sigma_{j}^{x}+\sum_{i} h_{i} \sigma_{j}^{x},
$$

with next-neighbor couplings $J_{i j} \in[-J, J]$ and local magnetic fields $h_{i} \in[-h, h]$ drawn randomly from uniform 


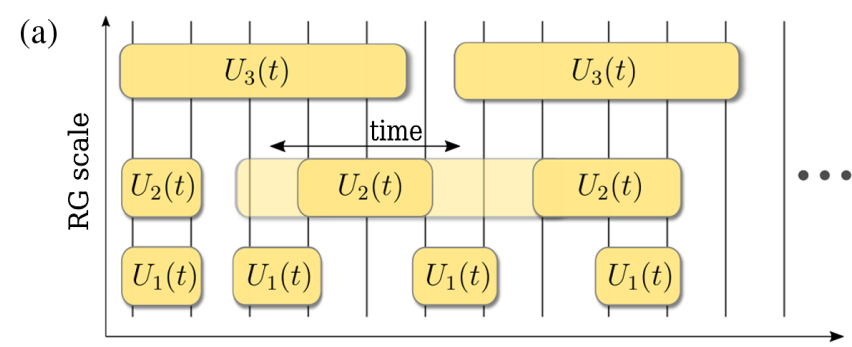

(b)

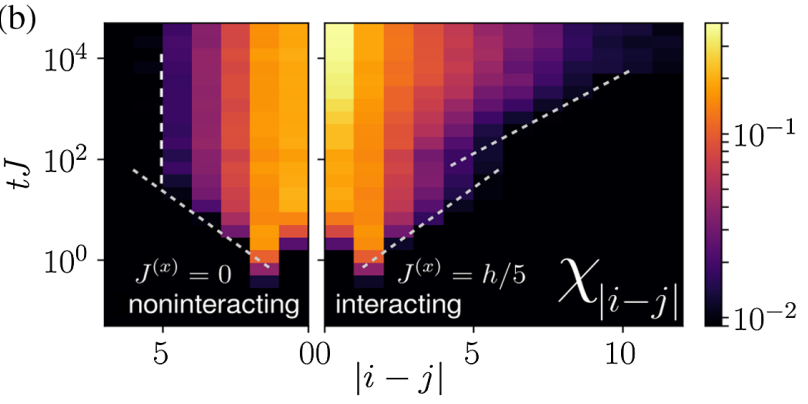

FIG. 1. (a) Illustration of random quantum circuit built up from local unitary RG transformations. In the course of the RG, longdistance and higher-order couplings emerge. Adding time dependence leads to further broadening with increasing time. (b) Spatiotemporal buildup of MBL spin-glass order in a random quantum Ising chain with 64 lattice sites after quenching a paramagnetic initial condition into the symmetry-broken phase at $J=5 h$ and $J^{(x)}=h / 5$ (interacting case). Dashed lines indicate emergence of light cones, except for noninteracting case $J^{(x)}=0$, where the order stops developing at a finite distance. Numerical data obtained from average over 25 disorder realizations.

distributions. We use periodic boundary conditions. For the 1D case, we also add random transverse couplings $J_{i j}^{(x)} \in$ $\left[-J^{(x)}, J^{(x)}\right]$ to obtain a generic and interacting model.

Solving the time evolution.-Before describing the utilized renormalization procedure and the training of the ANN in detail, let us start by outlining the general scheme for solving quantum real-time evolution utilizing strongdisorder RGs. Such an RG generates a sequence of local unitary transformations $U_{k}$ in order to iteratively obtain a simplified effective description of the considered quantum many-body system. In the time domain, we will show that this leads to the following representation of time-evolved quantum many-body states:

$$
|\psi(t)\rangle_{\mathrm{QC}}=e^{-i H_{0}^{(n)} t} U_{1}(t) \ldots U_{n}(t)\left|\psi_{0}\right\rangle,
$$

where a time dependence is added to the RG transformations $U_{k}$ through a generalized interaction picture; see below. The above equation maps quantum dynamics onto a quantum circuit generated by the local unitaries $U_{k}(t)$, where we use the notion of local when the support covers only a finite number of lattice sites. As the effective description in terms of the final Hamiltonian $H_{0}^{(n)}$ after the end of the RG procedure can be solved exactly, the complexity of the quantum circuit emerges solely from the unitaries $U_{k}(t)$. We find that such quantum circuits can become a nonperturbative object because the spatial support of the $U_{k}(t)$ typically grows over time, developing long-distance and higher-order couplings with large overlaps; see Fig. 1(a). A central contribution of this work is to outline a numerically exact scheme to encode $|\psi(t)\rangle_{\mathrm{QC}}$, and therefore the RG transformation itself, into an ANN using machine-learning techniques. The numerical learning effort and memory required in obtaining $|\psi(t)\rangle_{\mathrm{QC}}$ scales quadratically with system size while being independent of the spatial dimension.

Dynamical strong-disorder renormalization group.-In principle, quantum circuits such as in Eq. (2) can be generated using a variety of standard SDRGs. In the following, we introduce a variant of an SDRG, which we find improves the quantitative accuracy of the resulting scheme.

Like other SDRGs, the dynamical variant we introduce is based on a local separation of energy scales. Consequently, at the beginning of each iteration $k$, we pick the strongest coupling (also called the "fast mode") whose corresponding term in the Hamiltonian we call $H_{0}$. For the first iteration, this could be either a spin interaction $J_{i j}\left(J_{i j}^{(x)}\right)$ or a transverse field $h_{i}$; see Eq. (1). Those terms in the Hamiltonian that are not commuting with $H_{0}$ we denote by $V$. These can be eliminated perturbatively using a Schrieffer-Wolff transformation (SWT) [36] by applying a unitary transformation $W_{k}=e^{S_{k}}$ on the Hamiltonian with a generator $S_{k}$ satisfying $\left[H_{0}, S_{k}\right]=V$ and $S_{k}^{\dagger}=-S_{k}$ [12] at the expense of the renormalization $H_{0} \mapsto H_{0}+\left[S_{k}, V\right] / 2$. In general, this modifies existing couplings and leads to the generation of new terms in the Hamiltonian. After the SWT, the fast mode is decoupled from the remainder and can then be faithfully removed from the system as a second-order local integral of motion (LIOM) [37-39]. After $n$ such iterations, an unperturbed Hamiltonian $H_{0}^{(n)}$ is obtained, which is formed by the set of LIOMs.

For a general Hamiltonian, the types of newly generated couplings after each iteration are, of course, not known a priori. We approach this problem by representing at each stage of the RG the Hamiltonian as a sum of arbitrary Pauli strings $\sigma_{l_{1}}^{\alpha_{1}}, \ldots, \sigma_{l_{M}}^{\alpha_{M}}$ with a real coefficient $\lambda_{l_{1}, \ldots, l_{M}}$ each. Certainly, this approach can entail a costly handling of numerous generated higher-order couplings (see below), but it opens the possibility to take into account many-body resonances, which are neglected using earlier SDRGs $[11,40]$ and related so-called flow equation approaches $[34,41,42]$.

In addition, the accuracy of the RG can be further increased by splitting the SWT into infinitesimal unitary transformations, closely resembling in spirit the flow equation framework. This turns out to be particularly 
helpful in the vicinity of a critical point $(h \approx J$ here for the 1D model) where the SWT is least controlled. For a detailed presentation of the technical details, see the Supplemental Material [43]. To control the exponential number of couplings $\left\{V_{i}\right\}$ generated during the RG, we first neglect those terms where $\left|V_{i}\right| \ll t^{*-1}$, which are much smaller than the inverse of the targeted timescale $t^{*}$ and therefore do not influence physics up to timescales of $t^{*}$. Second, we perform the continuous renormalization only with respect to those $V_{i}$ whose relative magnitude lies above a fixed threshold, $\left|V_{i}\right| /\left|H_{0}\right|>\epsilon \ll 1$. Therefore, we have a tradeoff that is controlled by $\epsilon$ between exactness and the total number of couplings within the RG generators $S_{k}$ and the renormalized Hamiltonian $H_{0}^{(n)}$. In our computations, $\epsilon$ typically ranges from $10^{-4}$ to $10^{-2}$, depending on the closeness to the critical point $h \approx J$ or the ergodic transition $J^{(x)} \approx J$. Later, we present a quantitative analysis of our RG with respect to the dynamics of local observables.

Time-dependent unitaries. - To derive the time dependence of $U_{k}(t)$, we express the time-evolution operator in the renormalized basis,

$$
\begin{aligned}
e^{-i H t} & =e^{S_{1}^{\dagger}} \cdots e^{S_{n}^{\dagger}} e^{-i H_{0}^{(n)} t} e^{S_{n}} \cdots e^{S_{1}} \\
& =e^{-i H_{0}^{(n)} t} e^{S_{1}^{\dagger}(t)} \cdots e^{S_{n}^{\dagger}(t)} e^{S_{n}} \cdots e^{S_{1}} .
\end{aligned}
$$

We achieve a much more robust learning of the ANN upon successively commuting each factor $e^{S_{k}}$ to the left until its counterpart $e^{S_{k}^{\dagger}(t)}$ is reached. Identifying $U_{k}(t)=e^{\tilde{S}_{k}^{\dagger}(t)} e^{\tilde{S}_{k}}$ then gives the desired form as in Eq. (2). Here, $\tilde{S}_{k}$ denotes the total application of all rotations from $e^{S_{l}}(l<k)$ on $S_{k}$; see [43] for details.

Training the artificial neural network.-Utilizing ANNs as a variational ansatz for many-body wave functions has seen an active development recently [29,48], becoming competitive with or partially even superior to other state-ofthe-art methods $[30,49,50]$. In contrast to the commonly used time-dependent variational principle, which requires exponential efforts for reaching exponentially long times, we train the network with the unitaries $U_{1}(t), \ldots, U_{n}(t)$ during $n$ iterations (rows in the quantum circuit) to directly obtain $|\psi(t)\rangle_{\mathrm{QC}}$. Because the $U_{k}(t)$ are still local operators with a finite support in real space, we perform for each iteration $k$ a supervised learning procedure to find the set of complex network parameters $\tilde{\mathcal{W}}^{(k)}$ that minimizes the Fubini-Study metrics, given by $L\left[\tilde{\mathcal{W}}^{(k)}\right]^{2}=\arccos ^{2} y \approx$ $1-y^{2}$, with $y=\left|\left\langle\psi_{\tilde{\mathcal{W}}^{(k)}}\left|U_{k}(t)\right| \psi_{\mathcal{W}^{(k)}}\right\rangle\right|$; whereas

$$
\left|\psi_{\mathcal{W}}\right\rangle=\sum_{\{\vec{s}\}} \exp \left[H_{\mathrm{ANN}}(\mathcal{W}, \vec{s})\right]|\vec{s}\rangle
$$

refers to a quantum state defined by the output of an ANN, and $\{\vec{s}\}$ denotes the set of all spin configurations $\vec{s}=\left(s_{1}, s_{2}, \ldots\right), s_{i}= \pm 1$. Notice that we always assume properly normalized wave functions. Inserting the above definition of $\left|\psi_{\mathcal{W}}\right\rangle$ into $y$ results in a sum over all spin configurations. This sum can be efficiently sampled by the Markov chain Monte Carlo method [43]. Regarding the $U_{k}(t)$, we obtain them efficiently from the $\tilde{S}_{k}(t)$ again by utilizing the Pauli-string representation [43]. After convergence, the "learned" solution $\tilde{\mathcal{W}}^{(k)}$ is passed to the next iteration as $\mathcal{W}^{(k+1)}$. The network $H_{\mathrm{ANN}}(\mathcal{W}, \vec{s})$ can be considered as a deep forward extension of a complexvalued RBM with up to three hidden layers; see [43] for details.

Benchmarking.-In order to quantify the overall accuracy of our approach, we first benchmark the RG component and the machine-learning part individually. For the former task, we calculate Eq. (2) for small system sizes exactly using a matrix representation of the quantum circuit. Figure 2(a) shows a comparison of the local magnetization with the result obtained from exact diagonalization for a system of $L=12$ spins. The plot reveals that the accuracy of the dynamics depends crucially on the inclusion of many-body resonances, which is tuned by the only free RG parameter $\epsilon$; see above. For practical purposes, we set $\epsilon$ indirectly by imposing a maximum total number $\mathfrak{n}$ of couplings within all RG generators $S_{k}$. Here, $\mathfrak{n}=3(10) L$ corresponds to the label of excluded (included) many-body resonances and matches (exceeds) the number of original couplings. Already for $\mathfrak{n}=10 \mathrm{~L}$, we
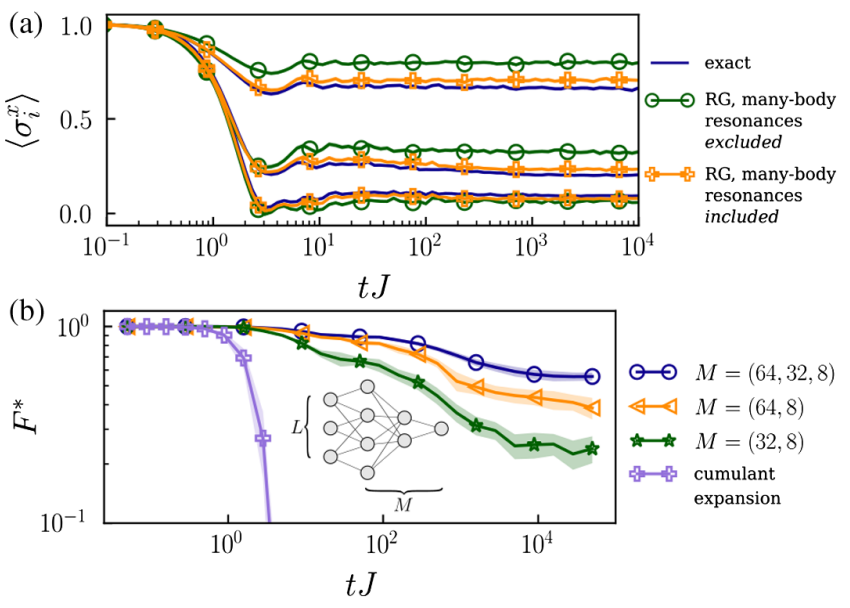

FIG. 2. (a) Comparing dynamics of transverse magnetization to exact diagonalization, averaged over lattice sites and 250 disorder realizations, with and without treatment of many-body resonances. Here, $L=12$ and $h=\{4,1,1 / 4\} J$ from top to bottom, and $J^{(x)}=J / 8$. (b) Lower bound $F^{*}$ on many-body fidelity, using $L=64$, of trained ANN state with state given by hypothetical, exact application of quantum circuit for different numbers of hidden units $M$ in each layer of deep forward ANN. System parameters are $J=5 h$ and $J^{(x)}=h / 5$ (interacting case). Shaded areas indicate uncertainties due to finite disorder ensemble of 25 realizations. Result from first-order cumulant expansion of quantum circuit shown for comparison. 


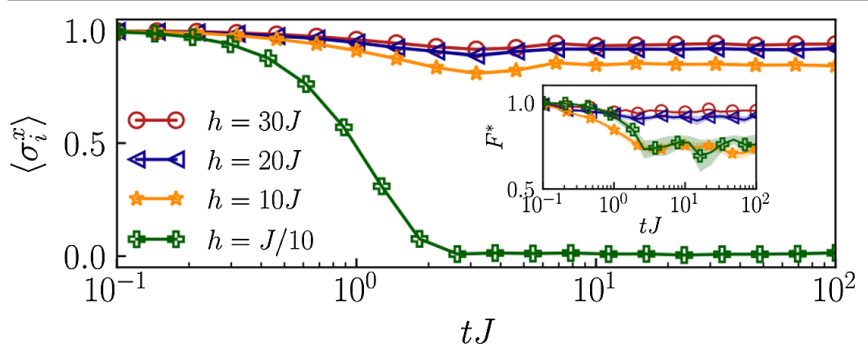

FIG. 3. Quench dynamics on $L=12 \times 12$ periodic simple square lattice with disordered next-neighbor Ising couplings and disordered transverse field. Local magnetization along initial direction of polarization plotted as average over lattice sites and 25 disorder realizations for various bounds of external field strengths. Inset shows lower bound of many-body fidelity as stated in Fig. 2(b).

observe a very good agreement, even for the longest times. Importantly, the result can be systematically improved by increasing $\mathfrak{n}$.

For benchmarking the ANN, we ideally would like to check the overlap $F=\left|\left\langle\psi_{\mathcal{W}^{(n)}}(t) \mid \psi_{\mathrm{QC}}(t)\right\rangle\right|$ of the final ANN state to the one obtained from an exact application of the quantum circuit, which is impossible for large system sizes. Nevertheless, we can offer a lower bound

$$
F^{*}=\prod_{k} F_{k}^{*}<F
$$

where $F_{k}^{*}=\left|\left\langle\psi_{\mathcal{W}^{(k+1)}}(t)\left|U^{(k)}(t)\right| \psi_{\mathcal{W}^{(k)}}(t)\right\rangle\right|$ denotes the partial overlaps measured at the end of each iteration $k$, which are byproducts of the training procedure. We plot $F^{*}$ in Fig. 2(b) as a function of time for the interacting 1D case and in the inset of Fig. 3 for a 2D lattice. It shows a high, macroscopic overlap, even for large system sizes, and a systematic improvement on adding more units and hidden layers to the ANN. From this finding, we conclude that the quantum circuit can be applied essentially numerically exactly on the ANN. For comparison, we also plot the result of a perturbative treatment,

$$
\left|\psi_{\mathrm{QC}}(t)\right\rangle \approx e^{-i H_{0}^{(n)} t} \sum_{\vec{s}} \prod_{k} \exp \left(\left\langle\vec{s}\left|S_{k}-S_{k}(t)\right| \psi_{0}\right\rangle\right)|\vec{s}\rangle,
$$

in Fig. 2(b) (i.e., a cumulant expansion of the quantum circuit) that neglects (higher-order) commutators between different $S_{k}$. It shows a rapid decay, and thus confirms the circuit's nonperturbative nature. In the Supplemental Material [43], we show further benchmarks of the whole framework for a large integrable system.

Let us add a few remarks on our choice of ANNs and the overall computational effort. Although ANNs are by definition universal function approximators (with stricter conditions in the complex case [51]), we most importantly demonstrate their practical ability to efficiently represent the relevant physical information of long-time-evolved wave functions. It might also be interesting to use tensor networks for the quantum circuit encoding but, there, the central challenge remains to represent volume-law entanglement for large systems and long times using reasonable bond dimensions [52].

The computational bottleneck of the overall procedure is clearly in the training process of the ANN. We estimate an ANN forward pass to effectively scale between $\mathcal{O}(L)$ and $\mathcal{O}\left(L^{2}\right)$, depending on whether the network structure exhibits short-range or long-range interconnections, respectively. With $L$ evaluations for a MC sweep, this results in a total scaling between $\mathcal{O}\left(L^{2}\right)$ and $\mathcal{O}\left(L^{3}\right)$. In contrast, the RG scales only linearly with $L$ (regarding time and memory) since the number of LIOMs is controlled by $\mathfrak{n} \propto L$. In practice, thanks to the Pauli-string representation being much more efficient than sparse matrices here, the RG always runs orders of magnitude faster than the training. Memory requirements of both parts are negligible compared to the resources available today.

Numerics. - As an application of our framework, we now explore nonequilibrium dynamics involving global quenches that has been difficult to access so far in the large system size and long-time limit. It is known from previous RG studies that a symmetry-broken state will keep a nonzero Edwards-Anderson order parameter in the longtime limit starting from symmetry-broken states if the system is in the MBL-spin-glass (MBL-SG) phase [40]. Here, we aim to address the buildup of spatiotemporal order starting from a $\mathbb{Z}_{2}$-symmetric state upon quenching into the MBL-SG phase. We detect the spatiotemporal dynamics of the MBL-SG order via [53]

$$
\chi_{i j}(t)=\sum_{\nu=1}^{4} p_{i j}^{(\nu)}(t)\left\langle e_{i j}^{(\nu)}(t)\left|\sigma_{i}^{z} \sigma_{j}^{z}\right| \varrho_{i j}^{(\nu)}(t)\right\rangle^{2},
$$

where $Q_{i j}$ denotes the reduced density matrix of two lattice sites $i$ and $j$, whereas $\nu$ enumerates its four eigenvectors $\left|\varrho_{i j}\right\rangle^{(\nu)}$ and eigenvalues (probabilities) $p_{i j}^{(\nu)}$. Fixing a distance $|i-j|$, we average $\chi_{i j}(t)$ across all associated pairs and disorder realizations. This quantity can be interpreted as a local version of the Edwards-Anderson order parameter, which is otherwise mostly used to detect MBL-SG order in a static context, but which does not exhibit a natural extension to the dynamical regime considered here.

Figure 1(b) shows $\chi_{d}(t)$ for both an interacting MBL $\left(J^{(x)}=h / 5\right)$ and a noninteracting Anderson localized $\left(J^{(x)}=0\right)$ case for a 1D chain of 64 spins. At short times of $t J \tilde{\leq} J / J^{(x)}=25$, an almost identical light cone for the buildup of MBL-SG correlations is visible, which appears consistent with a logarithmic growth. On longer timescales, we observe a fundamental difference between the Anderson and MBL cases. For the noninteracting Anderson-localized limit, the growth of MBL-SG order stops; whereas for $J^{(x)}>0$, a second light cone arises at a timescale that we 
estimate as $\sim 1 / J^{(x)}$. Interestingly, we find that all light cones do not become more open as we quench deeper into the MBL-SG phase but the more close we quench to the critical point. This behavior is reminiscent of the $l$-bit picture (see below), where LIOMs become more extended on approaching criticality. Right at criticality, $J=h$, even without interaction; we find that the order becomes genuinely long range as it decays algebraically with distance within the light cone. For the interacting case, inside the spin-glass phase, we observe an exponential decay with distance but with an essential difference from the noninteracting case: the order at any fixed distance does not saturate but increases strictly monotonically for all observed times within the light cones. This is a drastic nonperturbative effect of the interacting model. It is particularly obvious for next-neighboring spins; see Fig. 1(b). The important question of whether this growing will eventually lead to a finite plateau for $|i-j| \rightarrow \infty$ requires access to even much later times, which we currently cannot access.

When initializing the system in a symmetry-broken state, as studied in previous works, the stability of MBL-SG order originates from the large overlap with the LIOMs. The mechanism for the buildup of long-range order from symmetric states as targeted in this work is of fundamentally different origin because the initial state is oriented orthogonal to the LIOMs. Here, it is essential to generate long-distance quantum correlations between LIOMs. This is not possible in the Anderson localized limit because the LIOMs are independent, as we also see from our results in Fig. 1. Only in the interacting MBL limit can the MBL-SG order develop. Quantum correlations between two lattice sites $i$ and $j$ can emerge on a timescale $\left[J^{(x)}\right]^{-1} e^{|i-j| / \xi}$, where $\xi$ denotes a typical localization length. Consequently, at a given time $t$, the MBL-SG order can be generated over distances of $d \sim \xi \log \left[J^{(x)} t\right]$, explaining the appearance of the logarithmic light cone in Fig. 1(b).

As a closing point, we now turn briefly to quantum many-body dynamics in two dimensions. Whether a nonergodic phase due to strong disorder exists there has remained an outstanding challenge [54]. Its difficulty originates from the percolation of many-body resonances $[55,56]$. We find that, at least for sufficiently small or large external fields, the latter can be effectively captured using our framework up to an unprecedented long timescale. Figure 3 shows the temporal evolution of the local magnetization in a periodic simple square lattice, using essentially the same quench protocol as above. In contrast to the glassy dynamics of a chain, the lattice exhibits a rapid decay of magnetization at $h \ll J$, which is consistent with thermalization; see Fig. 3. On the other hand, for $h \gg J$, a stable nonthermal plateau is reached. Our result therefore numerically confirms a presumed quasilocalization $[55,56]$ in the disordered 2D transverse-field Ising model at infinite temperature.
Conclusion.-We have demonstrated how many-body quantum dynamics can be simulated for generic spin- $1 / 2$ systems up to exponentially long times given that sufficiently strong disorder breaks ergodicity at least up to the targeted timescale. Importantly, this includes an unbiased treatment of many-body resonances, which allowed us to obtain quantitative results in general and to go beyond onedimensional systems. We could show that our proposed framework does not fundamentally rely on any specific details of the model and scales up to system sizes far beyond what is possible with exact diagonalization. This opens up broad investigations, e.g., of nonthermal behavior and quantum aging dynamics in higher dimensions [57,58], long-range interacting systems [59-61], or localization in lattice gauge theories [62]. Since this work has shown that deep ANNs are able to apply the proposed quantum circuit numerically exact, the ansatz could also be well suited for random unitary circuit models, e.g., to study operator spreading [63-65] or measurement induced localization transitions [66,67].

We are grateful to M. Schmitt, M. Schiró, G. De Tomasi, and M. Schulz for helpful discussions. This project has received funding from the European Research Council under the European Union's Horizon 2020 Research and Innovation Programme (Grant Agreement No. 853443), and M. H. further acknowledges support by the Deutsche Forschungsgemeinschaft via the Gottfried Wilhelm Leibniz Prize program. Moreover, the authors gratefully acknowledge the Gauss Centre for Supercomputing e.V. (GCS; www.gauss-centre.eu) for funding this project by providing computing time through the John von Neumann Institute for Computing on the GCS Supercomputer JUWELS at the Jülich Supercomputing Centre.

*burau@pks.mpg.de

[1] P. W. Anderson, Science 177, 393 (1972).

[2] P. W. Anderson, Phys. Rev. 109, 1492 (1958).

[3] D. M. Basko, I. L. Aleiner, and B. L. Altshuler, Ann. Phys. (Amsterdam) 321, 1126 (2006).

[4] I. V. Gornyi, A. D. Mirlin, and D. G. Polyakov, Phys. Rev. Lett. 95, 206603 (2005).

[5] A. Pal and D. A. Huse, Phys. Rev. B 82, 174411 (2010).

[6] V. Oganesyan and D. A. Huse, Phys. Rev. B 75, 155111 (2007).

[7] R. Nandkishore and D. A. Huse, Annu. Rev. Condens. Matter Phys. 6, 15 (2015).

[8] J. Smith, A. Lee, P. Richerme, B. Neyenhuis, P. W. Hess, P. Hauke, M. Heyl, D. A. Huse, and C. Monroe, Nat. Phys. 12, 907 (2016).

[9] I. V. Protopopov, R. K. Panda, T. Parolini, A. Scardicchio, E. Demler, and D. A. Abanin, Phys. Rev. X 10, 011025 (2020).

[10] M. Schreiber, S. S. Hodgman, P. Bordia, H. P. Lüschen, M. H. Fischer, R. Vosk, E. Altman, U. Schneider, and I. Bloch, Science 349, 842 (2015). 
[11] R. Vosk and E. Altman, Phys. Rev. Lett. 110, 067204 (2013).

[12] D. Pekker, G. Refael, E. Altman, E. Demler, and V. Oganesyan, Phys. Rev. X 4, 011052 (2014).

[13] E. Altman and R. Vosk, Annu. Rev. Condens. Matter Phys. 6, 383 (2015).

[14] A. Morningstar and D. A. Huse, Phys. Rev. B 99, 224205 (2019).

[15] P. T. Dumitrescu, A. Goremykina, S. A. Parameswaran, M. Serbyn, and R. Vasseur, Phys. Rev. B 99, 094205 (2019).

[16] A. Goremykina, R. Vasseur, and M. Serbyn, Phys. Rev. Lett. 122, 040601 (2019).

[17] M. Žnidarič, T. Prosen, and P. Prelovšek, Phys. Rev. B 77, 064426 (2008).

[18] G. Vidal, Phys. Rev. Lett. 93, 040502 (2004).

[19] G. Vidal, Phys. Rev. Lett. 91, 147902 (2003).

[20] A. J. Daley, C. Kollath, U. Schollwöck, and G. Vidal, J. Stat. Mech. (2004) P04005.

[21] S. R. White and A. E. Feiguin, Phys. Rev. Lett. 93, 076401 (2004).

[22] F. Verstraete, J. J. Garcia-Ripoll, and J. I. Cirac, Phys. Rev. Lett. 93, 207204 (2004).

[23] M. Zwolak and G. Vidal, Phys. Rev. Lett. 93, 207205 (2004).

[24] S. Paeckel, T. Köhler, A. Swoboda, S. R. Manmana, U. Schollwöck, and C. Hubig, Ann. Phys. (Amsterdam) 411, 167998 (2019).

[25] H. Lin, Phys. Rev. B 42, 6561 (1990).

[26] D. J. Luitz, N. Laflorencie, and F. Alet, Phys. Rev. B 93, 060201(R) (2016).

[27] C. Kollath, A. M. Läuchli, and E. Altman, Phys. Rev. Lett. 98, 180601 (2007).

[28] J. Richter, T. Heitmann, and R. Steinigeweg, SciPost Phys. 9, 031 (2020).

[29] G. Carleo and M. Troyer, Science 355, 602 (2017).

[30] M. Schmitt and M. Heyl, Phys. Rev. Lett. 125, 100503 (2020).

[31] M. Schmitt and M. Heyl, SciPost Phys. 4, 013 (2018).

[32] E. Guardado-Sanchez, P. T. Brown, D. Mitra, T. Devakul, D. A. Huse, P. Schauß, and W. S. Bakr, Phys. Rev. X 8, 021069 (2018).

[33] G. De Tomasi, F. Pollmann, and M. Heyl, Phys. Rev. B 99, 241114(R) (2019).

[34] A. Hackl and S. Kehrein, Phys. Rev. B 78, 092303 (2008).

[35] D. S. Fisher, Phys. Rev. B 51, 6411 (1995).

[36] S. Bravyi, D. P. DiVincenzo, and D. Loss, Ann. Phys. (Amsterdam) 326, 2793 (2011).

[37] V. Ros, M. Müller, and A. Scardicchio, Nucl. Phys. B891, 420 (2015).

[38] J. Z. Imbrie, V. Ros, and A. Scardicchio, Ann. Phys. (Berlin) 529, 1600278 (2017).

[39] L. Rademaker and M. Ortuno, Phys. Rev. Lett. 116, 010404 (2016).

[40] R. Vosk and E. Altman, Phys. Rev. Lett. 112, 217204 (2014).
[41] S. J. Thomson and M. Schiró, Phys. Rev. B 97, 060201(R) (2018).

[42] S. J. Thomson and M. Schiró, Eur. Phys. J. B 93, 22 (2020).

[43] See Supplemental Material at http://link.aps.org/ supplemental/10.1103/PhysRevLett.127.050601 for further benchmarks and technical aspects of the methodology, which include Refs. [44-47].

[44] J. Van Hemmen, Z. Phys. B Condens. Matter 38, 271 (1980).

[45] P. Calabrese, F. H. L. Essler, and M. Fagotti, Phys. Rev. Lett. 106, 227203 (2011).

[46] P. Coleman, Introduction to Many-Body Physics (Cambridge University Press, Cambridge, England, 2015).

[47] J. Chen, D. Zhou, Y. Tang, Z. Yang, and Q. Gu, arXiv: 1806.06763.

[48] K. Ryczko, D. A. Strubbe, and I. Tamblyn, Phys. Rev. A 100, 022512 (2019).

[49] M. Hibat-Allah, M. Ganahl, L. E. Hayward, R. G. Melko, and J. Carrasquilla, Phys. Rev. Research 2, 023358 (2020).

[50] A. Nagy and V. Savona, Phys. Rev. Lett. 122, 250501 (2019).

[51] F. Voigtlaender, arXiv:2012.03351.

[52] D.-L. Deng, X. Li, and S. DasSarma, Phys. Rev. X 7, 021021 (2017).

[53] Y. Javanmard, S. Bera, and M. Heyl, Phys. Rev. B 99, 144201 (2019).

[54] P. Bordia, H. Lüschen, S. Scherg, S. Gopalakrishnan, M. Knap, U. Schneider, and I. Bloch, Phys. Rev. X 7, 041047 (2017).

[55] W. De Roeck and J. Z. Imbrie, Phil. Trans. R. Soc. A 375, 20160422 (2017).

[56] F. Alet and N. Laflorencie, C.R. Phys. 19, 498 (2018).

[57] T. B. Wahl, A. Pal, and S. H. Simon, Nat. Phys. 15, 164 (2019).

[58] J.-Y. Choi, S. Hild, J. Zeiher, P. Schauß, A. Rubio-Abadal, T. Yefsah, V. Khemani, D. A. Huse, I. Bloch, and C. Gross, Science 352, 1547 (2016).

[59] J. Zeiher, J.-Y. Choi, A. Rubio-Abadal, T. Pohl, R. van Bijnen, I. Bloch, and C. Gross, Phys. Rev. X 7, 041063 (2017).

[60] G. Piccitto, B. Žunkovič, and A. Silva, Phys. Rev. B 100, 180402(R) (2019).

[61] P. Hauke and L. Tagliacozzo, Phys. Rev. Lett. 111, 207202 (2013).

[62] P. Karpov, R. Verdel, Y.-P. Huang, M. Schmitt, and M. Heyl, Phys. Rev. Lett. 126, 130401 (2021).

[63] A. Nahum, S. Vijay, and J. Haah, Phys. Rev. X 8, 021014 (2018).

[64] C. W. von Keyserlingk, T. Rakovszky, F. Pollmann, and S. L. Sondhi, Phys. Rev. X 8, 021013 (2018).

[65] V. Khemani, A. Vishwanath, and D. A. Huse, Phys. Rev. X 8, 031057 (2018).

[66] Y. Bao, S. Choi, and E. Altman, Phys. Rev. B 101, 104301 (2020).

[67] C.-M. Jian, Y.-Z. You, R. Vasseur, and A. W. W. Ludwig, Phys. Rev. B 101, 104302 (2020). 